crabs, which use these beaches for development and which the public frequents on Long Island, NY.

Methods.-Metals were assessed in egg (prespawning), embryo (early postspawning), and larvae (late postspawning) stages. At all sites, eggs were extracted from adult females to obtain a baseline contamination level (ie, metals are transferred during egg formation). Embryos and larvae were collected from the sediment of each beach. Sediment and water were collected from all sites, and metal levels were compared to organism samples. Metal concentrations in all samples were determined using inductively coupled plasma mass spectrometry (ICP-MS). Descriptive statistics are reported and differences in life stages were analyzed using a Kruskal-Wallis test.

Results.-All trace metals accumulated, but to varying degrees based on life stage and beach. Essential metals that are required for physiological function showed significant accumulation from egg to larval stages for most sites $(P<$ .001). Nonessential metals, which can have toxic effects, showed significant accumulation from egg to larval stages for most sites $(P<.001)$.

Conclusions. - For a given metal, early stages related to sediment and water concentrations at some sites. Thus, local abiotic conditions may affect the uptake rate of each metal. We showed that metal bioaccumulation occurred at concentrations naturally found in habitats and suggested factors other than local levels of a contaminant should be considered when addressing the danger to organisms, including humans, that heavily use these areas.

Funding: WMS Hultgren Grant (2015).

\section{The Benefits of Wilderness Programs for Cancer Patients and Survivors: A Literature Review}

S. Terez Malka ${ }^{1}$; Tyler Warmack ${ }^{2}$

${ }^{1}$ Harvard School of Medicine, Boston, MA, USA, ${ }^{2}$ University of North Carolina School of Medicine, Chapel Hill, NC, USA

Introduction.-Many organizations promote wilderness experiences for cancer patients and survivors. Data suggest that moderate-to-vigorous exercise may reduce fatigue and depression in adults undergoing cancer treatment. The psychology literature suggests additional benefits of nature-based programming, including increased self-esteem and decreased anxiety. However, rigorous studies of the impact of wilderness-based programs are lacking.

Objective.-To evaluate the impact of wilderness programs on cancer patients and survivors.

Methods.-We searched PubMed, MEDLINE, CINAHL, and the Cochrane Central Register of Controlled Trials through March 2016. Search terms included adventure or wilderness or nature; therapy, rehabilitation, or treatment; and cancer. We reviewed the bibliographies of relevant articles for additional citations. Two authors independently analyzed the results for relevance and study design. Systematic reviews and controlled trials were selected for inclusion.

Results.-Forty-nine articles were identified: 38 were not relevant and 7 were case reports. Four studies were identified that met inclusion criteria, including 1 systematic review and 3 controlled trials. Outcomes of the controlled trials were heterogeneous, precluding meta-analysis. Two studies analyzed the impact of wilderness therapy programming on the physical activity levels of participants. The other 2 studies explored self-reported psychological benefits. Analysis of the 4 articles suggests benefits to program participants, including an increase in regular physical activity and improved perception of self-esteem, body image, quality of life, and mood. No studies assessed physical health outcomes or impact on disease progression.

Conclusions. - There are few rigorous studies evaluating the impact of adventure and wilderness programming on cancer patients and survivors, and no studies exploring medical effects. Preliminary data suggest positive, though potentially transient, benefits, including an increase in exercise frequency and improved self-esteem and mood. Given the popularity of these programs and potential benefits, further research is indicated to quantify the medical and psychological impact of wilderness programming on cancer patients and survivors.

Phase 3 Efficacy and Safety Results of Sufentanil Sublingual 30 mcg Tablet for Management of Acute Traumatic Pain in Emergency Medicine

James Miner ${ }^{1}$; Harold Minkowitz ${ }^{2}$; Zubaid Rafique ${ }^{3}$; Karen DiDonato ${ }^{4}$; Pamela Palmer ${ }^{4}$
${ }^{1}$ Hennepin County Medical Center, Minneapolis, MN, USA, ${ }^{2}$ Memorial Hermann Memorial City Medical Center, Houston, TX, USA, ${ }^{3}$ Ben Taub General Hospital, Houston, TX, USA, ${ }^{4}$ AcelRx Pharmaceuticals, Redwood City, CA, USA

Introduction.-Opioids often are considered standard-of-care treatment for acute pain associated with field trauma; however, limitations of these therapies have been well documented. Morphine is associated with erratic onset of analgesia when delivered intramuscularly (IM) and delayed side effects resulting from active metabolites, and fentanyl's rapid distribution demands frequent redosing. There remains a clinical need for rapid-acting, potent analgesics that do not require an invasive route of delivery. In collaboration with the Department of Defense, a sufentanil sublingual $30 \mathrm{mcg}$ tablet (SST) is in development for treatment of acute pain in battlefield and emergency trauma settings.

Objective.-To evaluate SST's safety and efficacy in managing moderateto-severe pain in the emergency department (ED).

Methods.-This is a multicenter, open-label, phase 3 study in up to 120 adult patients presenting to the ED with acute traumatic pain. SST was administered as needed but not more frequently than hourly. Efficacy was assessed by patient reports of pain intensity on an 11-point numerical rating scale $(0=$ no pain and $10=$ worst possible pain $)$. The primary efficacy variable was the summed pain intensity difference from baseline over the first hour (SPID1). Safety was assessed via vital signs and adverse event reporting (AEs).

Results.-Forty of the 120 patients have been enrolled to date and an interim analysis performed. Statistically significant and clinically meaningful reductions in pain intensity have been observed following a single dose of SST A low number of adverse events has been reported, with nausea and somnolence the most frequent (5\% each). All available safety and efficacy results will be included at the time of presentation.

Conclusions.-Sufentanil $30 \mathrm{mcg}$ tablets are in development for treatment of moderate-to-severe acute pain in an EM population. Early efficacy and tolerability results suggest that SST may offer a viable alternative to IM or IV dosing.

Funding: The late-stage development of this drug is being sponsored in part by the US Department of Defense.

\section{An Assessment of Diarrhea Among Long-Distance Backpackers in the Sierra Nevada}

Derek Meyer $^{1}$; Amber Costantino ${ }^{2}$; Susanne Spano ${ }^{1}$

${ }^{1}$ UCSF - Fresno, Fresno, CA, USA, ${ }^{2}$ California State University -

Fresno, Fresno, CA, USA

Introduction.- The John Muir Trail (JMT) is a 210-plus mile, longdistance backpacking trail in the Sierra Nevada mountain range of California Previous studies from the Appalachian Trail and Long Trail have found significant correlation between backpacker demographics and on-trail hiking behaviors and diarrhea incidence, ranging from $10.7 \%$ to $63 \%$. Incidence of diarrhea also has been shown to depend on age, sex, and regularity of standard backcountry hygiene practices. However, no studies to date have examined these variables among backpackers on the JMT.

Objectives.-To determine the significance of particular JMT backpacker demographics and hygiene compliance regularity with diarrhea incidence and severity.

Methods. - Survey data from long-distance backpackers who attempted a JMT trek in 2014 were assessed via statistical software for the significance of variables that might contribute to the incidence and severity of on-trail diarrhea These variables included age, sex, prehike body weight, prehike physical activity (hours/week), prehike self-assessed physical condition, previous hiking or backpacking experience (days in previous 10 years), solo or group backpacking itinerary, on-trail average pack-weight, on-trail hygiene compliance regularity (water filtration, hand hygiene, dish washing), and posthike difficulty assessment. Backpackers also reported their on-trail incidence and severity of diarrhea.

Results.-Of 737 valid responders, $16.4 \%$ reported experiencing diarrhea ( $82 \%$ with minimal or mild severity; $18 \%$ with significant severity). No statistical significance was found between diarrhea incidence as related to hiker demographics or compliance with standard hygiene recommendations. Regular hand sanitizer use was significantly correlated with having more severe diarrhea, but 